\title{
Conserved contributions of NMDA receptor subtypes to synaptic responses in lamina II spinal neurons across early postnatal development
}

\author{
Hadir Mahmoud ${ }^{1,2}$, Newton Martin ${ }^{1}$ and Michael E. Hildebrand ${ }^{1,2,3^{*}}$ (1)
}

\begin{abstract}
NMDA receptors are heteromeric complexes that contribute to excitatory synaptic transmission and plasticity. The presence of specific variants of GluN2 subunits in these complexes enables diversity in NMDA receptor function and regulation. At brain synapses, there is a switch from slow GluN2B-mediated NMDA receptors to faster GluN2Adominated NMDA receptors as well as an increase in the ratio of AMPA to NMDA receptors during early postnatal development. This glutamate receptor switch is observed across brain regions and is critical for synaptic maturation, circuit development, and associative learning. However, whether a similar receptor subunit switch occurs within pain processing neurons in the developing spinal cord remains untested. To investigate this, we performed wholecell patch clamp recordings of excitatory synaptic responses from lamina II dorsal horn neurons of one to three week-old rats. We found that GluN2B and GluN2A both prominently contribute to NMDA receptor responses at neonatal lamina II synapses, with a small contribution from GluN2D as well. Surprisingly, we found that this molecular identity of NMDA receptor responses as well as the relative contribution of AMPA receptors versus NMDA receptors did not change at lamina II synapses across early postnatal development (P7 to P21). The lack of a developmental switch and persistence of slow-decaying GluN2B- and GluN2D-mediated synaptic responses throughout neuronal maturation in the dorsal horn has implications for understanding both the regulation of synaptic glutamatergic receptors as well as spinal mechanisms of pain processing.
\end{abstract}

Keywords: Spinal cord, Dorsal horn, Lamina II, Synapse, NMDA receptor, GluN2A, GluN2B, GluN2D, AMPA receptor, Development

\section{Introduction}

The superficial layers of the spinal cord dorsal horn, laminae I and II, are a hub for processing pain-related sensory inputs [1]. Disruption in the balance of excitability within the superficial dorsal horn can drive chronic pain [2], and yet the specific molecular determinants that mediate synaptic signaling across dorsal horn neuron subpopulations remain poorly understood. The $\mathrm{N}$-methyl-D-aspartate receptor (NMDAR) subtype of ionotropic glutamate receptors are critical mediators of

\footnotetext{
* Correspondence: mike.hildebrand@carleton.ca

${ }^{1}$ Department of Neuroscience, Carleton University, Ottawa, ON, Canada

${ }^{2}$ Neuroscience Program, Ottawa Hospital Research Institute, Ottawa, ON, Canada

Full list of author information is available at the end of the article
}

synaptic transmission and plasticity throughout the nervous system. Within the superficial dorsal horn, synaptic NMDARs are implicated in both physiological and pathological mechanisms of pain processing [3]. Of the seven variants of genetically encoded NMDAR subunits (GluN1, GluN2A-D, and GluN3A-B), the majority of functional NMDAR heteromeric complexes contain two obligatory GluN1 subunits and two identical or different GluN2 subunits. The identity of the GluN2 subunit(s) shapes both the functional properties and regulation of synaptic NMDAR responses. For example, GluN2A-, GluN2B/C-, and GluN2D-containing diheteromeric NMDARs have fast $(\sim 50 \mathrm{~ms})$, intermediate $(\sim 250 \mathrm{~ms})$, and slow (>1000 ms) deactivation rates, respectively [4]. While GluN2A-containing receptors dominate NMDAR

(c) The Author(s). 2020 Open Access This article is distributed under the terms of the Creative Commons Attribution 4.0 International License (http://creativecommons.org/licenses/by/4.0/), which permits unrestricted use, distribution, and 
responses at the majority of mature central synapses [4], synaptic NMDAR responses within the superficial dorsal horn can be mediated by GluN2A-, GluN2B- and/or GluN2D-containing NMDARs, depending on the specific synaptic input, laminae and developmental time point under study [5-7]. To gain a better understanding of the roles of individual NMDAR isoforms in dorsal horn nociceptive signalling, the biophysical and pharmacological properties of synaptic NMDARs need to be systematically studied and compared between distinct developmental stages for each subpopulation of superficial dorsal horn neuron.

In the first three weeks of postnatal brain development, there is a switch from predominant expression of GluN2Band GluN2D- containing NMDARs at birth to a progressive increase in the relative expression of GluN2Acontaining NMDARs [4]. The well-documented switch from GluN2B-mediated to GluN2A-mediated NMDAR responses at developing central synapses produces a dramatic decrease in NMDAR synaptic decay constants as well as an associated increase in the relative synaptic contribution of $\alpha$-amino-3-hydroxy-5-methyl-4-isoxazolepropionate receptors (AMPARs) compared to NMDARs $[8,9]$. The early dominance of slow deactivating GluN2B NMDARs promotes synaptic potentiation and maturation when there is correlated activity, while the subsequent switch to GluN2A dominance decreases synaptic strength and dampens further potentiation [10]. This synaptic switch is conserved across diverse brain regions and allows for maturation of associative learning abilities [11].

Our observation of GluN2B- and GluN2D-dominated NMDAR responses at a mature dorsal horn synapse [7] calls into question whether the canonical synaptic switch from GluN2B to GluN2A is conserved in the developing dorsal horn nociceptive network. Here, we use whole-cell patch clamp recordings in combination with subtype-specific GluN2 antagonists to systematically investigate excitatory glutamatergic responses and the contributions of GluN2 isoforms to the NMDAR component of responses at lamina II synapses across early postnatal spinal cord development.

\section{Results}

The biophysical properties of glutamatergic synaptic responses in lamina II neurons are conserved across early postnatal spinal cord development

To characterize postsynaptic glutamatergic responses at developing lamina II synapses, we performed whole-cell patchclamp recordings on lamina II neurons from acute transverse spinal sections of postnatal day 7 (P7) to 21 (P21) male rats. We recorded miniature excitatory postsynaptic currents (mEPSCs) at $-60 \mathrm{mV}$ and $+60 \mathrm{mV}$ to study AMPAR- and NMDAR-mediated postsynaptic responses, respectively [7]. In lamina II neurons held at $-60 \mathrm{mV}$, we observed inward AMPAR-mediated mEPSCs with a peak amplitude of -
$15.9 \pm 0.9 \mathrm{pA}$ and a decay constant $\left(\tau_{\text {decay }}\right)$ of $11.1 \pm 1.1 \mathrm{~ms}$ ( $n=40$ animals, 62 neurons, Fig. $1 \mathrm{a}$ ). At a holding potential of $+60 \mathrm{mV}$, outward mEPSCs were much slower than mEPSCs at $-60 \mathrm{mV}$ for the same neurons (Fig. 1a). To estimate the amplitude of the NMDAR component of mEPSCs at $+60 \mathrm{mV}$, we measured the mEPSC amplitude at $20 \mathrm{~ms}$ from onset, a timepoint of peak NMDAR response where the contribution from fast deactivating AMPARs is minimal [7]. The average amplitude of NMDAR mEPSCs at $+60 \mathrm{mV}$ was $16.3 \pm 0.7 \mathrm{pA}$, which was not significantly different from the absolute peak amplitude of mEPSCs at $-60 \mathrm{mV}(n=62$ neurons, $p=0.68)$. The decay constant for NMDAR mEPSCs at $+60 \mathrm{mV}$ $(169 \pm 7 \mathrm{~ms})$ was significantly slower than that for AMPAR mEPSCs at $-60 \mathrm{mV}\left(n=62, p=1.9 \mathrm{e}^{-31}\right.$, Fig. 1a). We next compared the biophysical properties of AMPAR and NMDAR mEPSCs at distinct time points in early postnatal development. Our developmental age bins of P7-P11, P12$\mathrm{P} 16$, and P17-P21 correspond to postnatal periods that encompass the complete synaptic switch process across brain regions [11] and also correspond to periods of maximal change in primary afferent-induced postsynaptic responses and plasticity for developing dorsal horn neurons [13]. We found that the ratio of the peak amplitude of AMPAR mEPSCs at $-60 \mathrm{mV}$ compared to the amplitude of NMDAR mEPSCs at $+60 \mathrm{mV}$ (at $20 \mathrm{~ms}$ ) remained unchanged $(p=0.67)$ near a value of 1 across the three postnatal time periods (P7-P11, $1.1 \pm 0.1, n=22$ neurons; P12P16, $1.0 \pm 0.1, n=19$; P17-P21, $1.1 \pm 0.1, n=21$; Fig. 1b) We therefore conclude that AMPARs and NMDARs contribute equally to excitatory synaptic responses in lamina II neurons across early postnatal rodent development.

To further investigate the NMDAR component of lamina II synaptic responses across early development (Fig. 1c), we calculated the charge transfer through mEPSCs at $+60 \mathrm{mV}$ from $20 \mathrm{~ms}$ to $500 \mathrm{~ms}$ after event onset. Synaptic charge transfer during this time range is exclusively mediated by NMDAR and not AMPAR activity [7]. We found that there was no significant difference $(p=0.42)$ in the magnitude of NMDAR charge transfer between P7-P11 $(n=22)$, P12-P16 $(n=19)$, and P17-P21 $(n=21$; Fig. 1d). Similarly, there was no significant change in decay constants for NMDAR mEPSCs at lamina II synapses across early postnatal development (Fig. 1c, e). Taken together, our results suggest that the relative contribution and biophysical properties of synaptic NMDARs do not change during the postnatal maturation of lamina II spinal cord neurons.

\section{Both GluN2B- and GluN2A-containing NMDARs mediate lamina II synaptic responses across early postnatal development}

The lack of change in charge transfer and decay constants of NMDAR mEPSCs in developing lamina II 


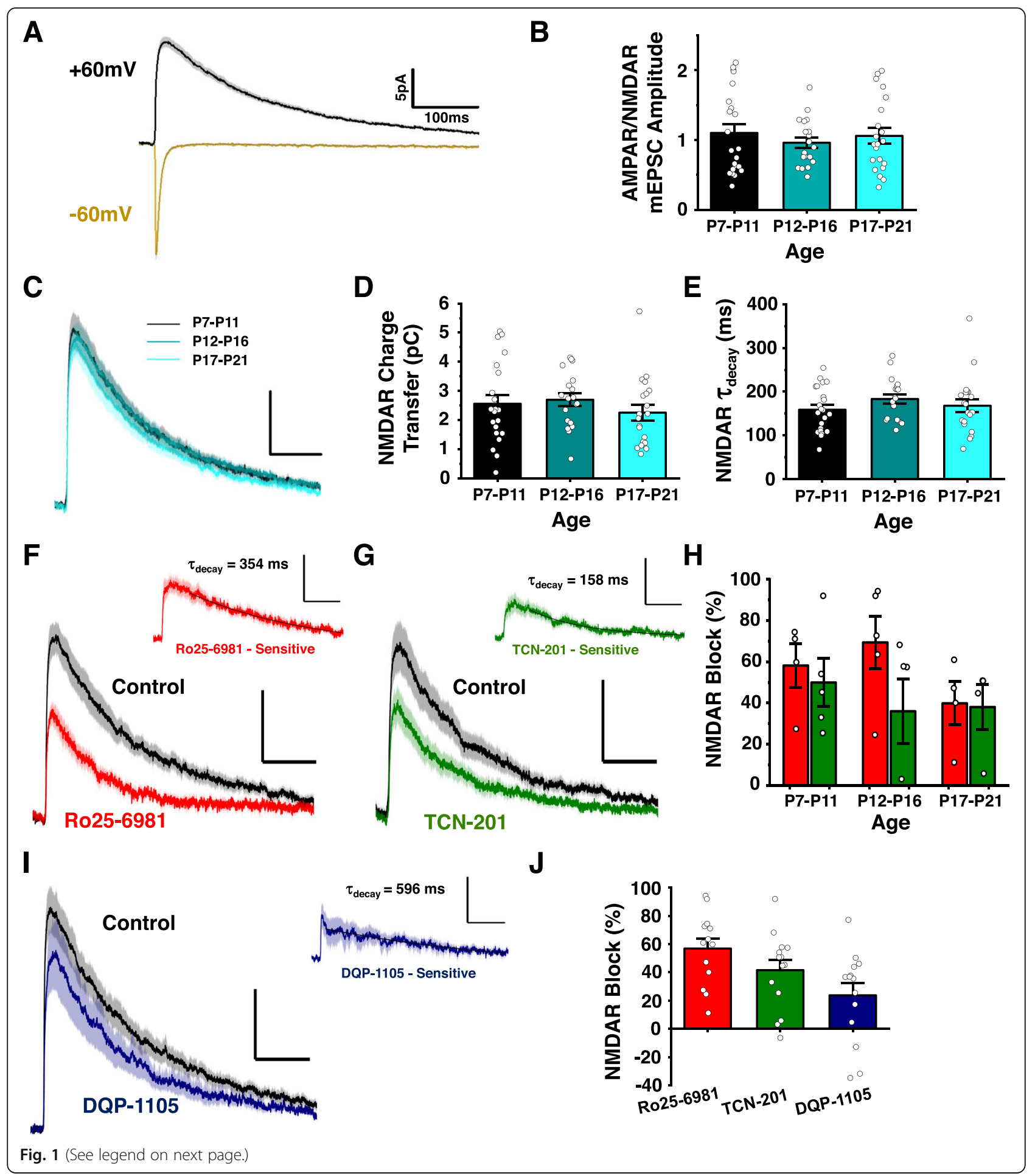




\section{(See figure on previous page.)}

Fig. 1 The biophysical and pharmacological properties of AMPAR- and NMDAR-mediated synaptic responses in lamina II neurons do not change across early postnatal development. (A) Averaged mEPSC traces of lamina II neurons ( $n=62$ ) at both $-60 \mathrm{mV}$ (gold) and $+60 \mathrm{mV}$ (black) from early postnatal (P7-P21) male rats. (B) Ratio of mEPSC peak amplitude at $-60 \mathrm{mV}$ versus peak amplitude at $+60 \mathrm{mV}$ (at $20 \mathrm{~ms}$ from onset) from lamina II neurons of postnatal rats aged P7 to P11 (black, $n=22$ neurons), P12 to P16 (teal, $n=19$ neurons), and P17 to P21 (aqua, $n=21$ neurons) (C) Averaged mEPSC traces at $+60 \mathrm{mV}$ for rats and neurons in the same postnatal age bins described in B. (D) Charge transfer through the NMDAR component of mEPSCs at $+60 \mathrm{mV}$ (from 20 to $500 \mathrm{~ms}$ ). (E) The exponential decay constants measured from mEPSCs at $+60 \mathrm{mV}$. (F) Averaged mEPSCs at $+60 \mathrm{mV}$ before (black) and after (red) application of the GluN2B antagonist, 1 uM Ro25-6981 ( $n=13$ ). Inset, Ro25-6981sensitive NMDAR mEPSC difference current. (G) Averaged mEPSCs at $+60 \mathrm{mV}$ before (black) and after (green) application of the GluN2A antagonist, 10 uM TCN-201 ( $n=14)$. Inset, TCN-201-sensitive mEPSC difference current. $(\mathbf{H})$ The percent reduction in NMDAR charge transfer by both 1 uM Ro25-6981 (red, left bars) and 10 uM TCN-201 (green, right bars) were not significantly different across the various developmental time points: P7-P11 $(n=4,5)$, P12-P16 ( $n=5,5)$, P17-21 $(n=4,4)$. (I) Averaged NMDAR mEPSCs before (black) and after (blue) application of 10 uM DQP-1 105 ( $n=14)$. Inset, 10 uM DQP-1105-sensitive NMDAR mEPSC difference current. (J) Percent NMDAR charge transfer reduction produced by administration of 1 uM Ro25-6981 (red, $n=13$ ), 10 uM TCN-201 (green, $n=14$ ) or 10 uM DQP-1105 (blue, $n=14$ ). Cells with a negative blockade reflect an antagonist-independent run-up in NMDAR charge transfer [12] during the course of recording. All traces are presented as mean (darker line) $+/-$ standard error (lighter shaded area). Scale bar $x$ axes $=100 \mathrm{~ms}$, y axes $=5$ pA

neurons suggests that there may not be an early postnatal switch from GluN2B-containing NMDARs to GluN2A-containing NMDARs at these specific central synapses. In fact, a stable decay constant between approximately $160 \mathrm{~ms}$ and $180 \mathrm{~ms}$ across lamina II development (Fig. 1e) suggests that both GluN2A- and GluN2B-containing NMDARs may mediate synaptic responses throughout the maturation process [4]. We therefore used GluN2 subunit-specific pharmacological antagonists to directly explore the relative contribution of individual GluN2 subtypes to NMDAR-mediated mEPSCs. The contribution of GluN2B was investigated using the GluN2B-specific antagonist, Ro25-6981, at a concentration $(1 \mu \mathrm{M})$ that inhibits recombinant GluN2Bbut not GluN2A-containing diheteromeric NMDARs [14] and that we have shown to selectively inhibit GluN2B-like NMDAR responses at lamina I synapses [7] (see Methods for further discussions on NMDAR antagonists). NMDAR charge transfer was calculated for a 10min baseline control recording period and compared to a time period where inhibition reached a steady-state level, typically after 40 minutes of drug administration. We found that administration of $1 \mu \mathrm{M}$ Ro25-6981 resulted in a significant block of NMDAR mEPSCs at P7P21 lamina II synapses $(56.9 \pm 7.1 \%, n=13, p=0.00035$, Fig. 1f). To test for the contribution of GluN2A to NMDAR-mediated mEPSCs, we utilized the GluN2Aspecific antagonist, TCN-201, at a concentration $(10 \mu \mathrm{M})$ that selectively blocks only GluN2A-containing recombinant NMDARs [15] and that inhibits fastdecaying GluN2A-like but not slower decaying GluN2Blike synaptic NMDAR responses at rat lamina I synapses [7]. In contrast to mature lamina I neurons [7], we found that administration of $10 \mu \mathrm{M}$ TCN-201 also robustly reduced NMDAR-mediated charge transfer at early postnatal lamina II synapses $(41.6 \pm 7.2 \%, n=14, p=0.00064$, Fig. 1g). There was no significant difference between the baseline NMDAR charge transfers for $1 \mu \mathrm{M}$ Ro25-6981and $10 \mu \mathrm{M}$ TCN-201-treated neurons $(p=0.90)$, suggesting that NMDAR biophysical properties did not diverge between lamina II neurons from the two treatment groups (Supplementary Fig. 1). To assess the GluN2 subunit-selectivity of the specific pharmacological antagonists, the drug-sensitive mEPSC difference current was graphed and fitted with an exponential decay constant (Fig. 1f, g). The $1 \mu \mathrm{M}$ Ro25-6981-sensitive difference current displayed a decay constant of 354 $\mathrm{ms}$ (Fig. 1f), which falls within the deactivation range for GluN2B-containing diheteromeric NMDARs [4, 7]. The $10 \mu \mathrm{M}$ TCN-201-sensitive difference current had a decay constant of $158 \mathrm{~ms}$ (Fig. 1g), consistent with the faster deactivation rate observed for GluN2A-containing NMDARs [4, 7]. From these findings, we conclude that synaptic NMDAR responses are primarily mediated by a combination of GluN2A- and GluN2B-containing receptors in P7 to P21 lamina II neurons.

We next explored whether the functional contribution of GluN2A versus GluN2B-containing receptors to synaptic responses changes during the postnatal maturation of lamina II neurons. To test this, we analyzed the effects of Ro25-6981 and TCN-201 on NMDAR charge transfer across the three distinct postnatal development periods: P7-P11 $\left(\mathrm{n}_{\mathrm{Ro}}=4, \mathrm{n}_{\mathrm{TCN}}=5\right)$, P12-P16 $\quad\left(\mathrm{n}_{\mathrm{Ro}}=5\right.$, $\left.\mathrm{n}_{\mathrm{TCN}}=5\right)$, and P17-P21 $\left(\mathrm{n}_{\mathrm{Ro}}=4, \mathrm{n}_{\mathrm{TCN}}=4\right)$. Two-way ANOVA analysis revealed no significant difference in the magnitude of NMDAR block for either $1 \mu \mathrm{M}$ Ro25$6891(p=0.24)$ or $10 \mu \mathrm{M}$ TCN-201 $(p=0.72)$ between the three postnatal development periods (Fig. 1h). We therefore conclude that the relative contribution of GluN2A versus GluN2B to synaptic NMDAR responses in lamina II neurons does not change across early postnatal development.

\section{GluN2D has a minor contribution to mEPSCs at early postnatal lamina II synapses}

Given that GluN2D-containing receptors prominently contribute to NMDAR responses at lamina I synapses [7], we next explored whether GluN2D NMDARs 
mediate a component of NMDAR mEPSCs at developing lamina II synapses. We utilized the GluN2Dselective antagonist, DQP-1105, at a concentration $(10 \mu \mathrm{M})$ that inhibited approximately $85 \%$ of GluN2D NMDARs with negligible effects on GluN2A or GluN2B NMDARs [16] and that also inhibited native GluN2Dlike synaptic NMDAR responses in lamina I neurons [7]. Treatment with $10 \mu \mathrm{M}$ DQP-1105 resulted in a $23.7 \pm$ $8.6 \%$ reduction in NMDAR charge transfer at P7-P21 lamina II synapses (Control $2.78 \pm 0.32 \mathrm{pC}, D Q P-1105$ $2.07 \pm 0.40 \mathrm{pC}, n=14, p=0.033$, Fig. 1i). The $10 \mu \mathrm{M}$ DQP-1105-sensitive mEPSC difference current exhibited a decay constant of $596 \mathrm{~ms}$ (Fig. 4A), which is consistent with slow deactivating GluN2D-containing NMDARs [4, 7]. We found that the NMDAR blockade by DQP-1105 at lamina II synapses was less than that produced by Ro25-6981 or TCN-201 (Fig. 1j).

\section{Discussion}

In this study, we used whole-cell patch-clamp recordings to show that spontaneous synaptic responses in lamina II neurons include a fast AMPAR-mediated component and a slower NMDAR-mediated component, which are equal in amplitude across early postnatal development. In contrast to mature lamina I neurons [7], we find that both GluN2A- and GluN2B-containing receptors prominently contribute to synaptic NMDAR responses in developing lamina II neurons, with a smaller contribution from GluN2D-containing receptors as well. Surprisingly, the roles of these three GluN2 NMDAR subunits in mediating lamina II synaptic responses remains constant across early postnatal development. We therefore conclude that unlike the vast majority of central synapses, there is no molecular switch in the composition of NMDARs and no change in the ratio between functional AMPARs and NMDARs at developing postnatal lamina II synapses.

Unlike in the brain [4], we found that the relative contribution of GluN2A- versus GluN2B-containing NMDARs to lamina II synaptic responses does not change between weeks one and three of postnatal development. This extends the initial pioneering work of Bardoni and colleagues demonstrating that the magnesium sensitivity and kinetics of synaptic NMDAR responses in laminae I and II neurons do not change in the first postnatal week of rat development [6]. The lack of the canonical downregulation of slow-decaying GluN2B- (and GluN2D-) mediated synaptic NMDARs for developing superficial dorsal horn neurons could potentially underlie the increased propensity for synaptic potentiation and central sensitization within these pain signaling circuits. Understanding the molecular differences in the developmental regulation of synaptic physiology and plasticity between the brain and spinal cord will therefore potentially inform the development of new therapeutic approaches for the treatment of chronic pain.

As NMDAR charge transfer and decay kinetics as well as blockade by specific GluN2 subunit antagonists varied considerably between individual lamina II neurons, the next step is to explore potential heterogeneity in the molecular composition of synaptic NMDARs between lamina II neuron subpopulations. The outer half of lamina II that we were recording from contains different types of excitatory and inhibitory nociceptive interneurons, which can be classified based on distinctions in morphology, excitability and neurochemistry [17], and, more recently, on RNA expression profiles [18, 19]. Future studies could combine biophysical and pharmacological characterization of synaptic NMDAR responses from individual lamina II neurons, as reported here, with neurochemical and morphological characterization of filled and labelled neurons. Moreover, single cell RNA sequencing from superficial dorsal horn tissue or even individual recorded neurons could be used to characterize the subtypes of NMDAR subunits that are functionally expressed within specific transcriptionally-identified subtypes of excitatory and inhibitory interneurons $[18,19]$. This information is essential for uncovering the differential roles of excitatory glutamate receptor subtypes in synaptic plasticity and nociceptive function in subpopulations of dorsal horn neurons across development.

\section{Methods \\ Animals}

Male Sprague Dawley rats aged between postnatal day 7 to 21 (P7-P21) were ordered from Charles River and used for all experiments. All procedures and experimental protocols followed the guidelines set out by the Canadian Council on Animal Care regarding animal care and handling. Animal research protocols were authorized by the Animal Care Committee at Carleton University and the Animal Veterinary Services at The Heart Institute, where animals were housed.

\section{Spinal cord isolation and tissue sectioning}

Early postnatal (P7-P21) male Sprague Dawley rats were anaesthetized using intraperitoneal injections of $20 \%$ (wt/vol) urethane (Sigma-Aldrich) at $0.1 \mathrm{~mL} / 10 \mathrm{~g}$ of weight. The spinal cord was excised immediately following anesthesia and placed in an ice-cold, bubbled (5\% $\mathrm{CO}_{2}, 95 \% \mathrm{O}_{2}$ ) sucrose cutting solution containing (in $\mathrm{mM}$ ): 50 sucrose, $92 \mathrm{NaCl}, 15 \mathrm{D}$-(+)-glucose, $26 \mathrm{NaCO}_{3}$, $5 \mathrm{KCl}, 1.25 \mathrm{NaH}_{2} \mathrm{PO}_{4}, 0.5 \mathrm{CaCl}_{2}, 7 \mathrm{MgSO}_{4}$, and $1 \mathrm{mM}$ kynurenic acid. The trimmed spinal segment containing L3-L6 was mounted on its ventral surface to a piece of agar, which was then glued vertically to a vibratome sectioning chuck. Transverse $(400 \mu \mathrm{m})$ slices were generated using a Leica VT1200S vibratome. Spinal cord 
tissue sections were placed into a bubbled sucrose cutting solution that did not contain kynurenic acid and was heated to $\sim 33.9^{\circ} \mathrm{C}$ using a water bath (Fisher-Scientific). The slices were allowed to recover for $40 \mathrm{~min}$ at this temperature before the solution chamber was removed from the water bath and allowed to passively reach room temperature $\left(\sim 23^{\circ} \mathrm{C}\right)$.

\section{Whole-cell patch-clamp electrophysiological recordings on lamina II spinal cord neurons}

Spinal cord sections were visualized under a Zeiss microscope and acutely perfused with artificial cerebral spinal fluid (aCSF) containing (in $\mathrm{mM}$ ): $125 \mathrm{NaCl}, 3$ $\mathrm{KCl}, 26 \mathrm{NaCO}_{3}, 1.25 \mathrm{NaH}_{2} \mathrm{PO}_{4}, 2 \mathrm{CaCl}_{2}, 1 \mathrm{MgCl}_{2}$, and $20 \mathrm{D}$-(+)-glucose, $10 \mu \mathrm{M}$ bicuculline (Tocris Bioscience), $10 \mu \mathrm{M}$ strychnine, $10 \mu \mathrm{M} \mathrm{Cd}^{2+}$, and $0.5 \mu \mathrm{M}$ TTX (Alomone Labs). Neurons located in the outer half of lamina II were visualized first under 10x based on their location in the substantia gelatinosa, followed by $40 \mathrm{x}$ magnification. Patch-clamp recording borosilicate glass pipettes of 5 to $11 \mathrm{M} \Omega$ resistance were pulled using a Sutter Flaming micropipette puller and fire-polished. Pipettes were filled with an internal solution containing (in mM): 105 D-gluconic acid, $105 \mathrm{CsOH}, 17.5 \mathrm{CsCl}, 10$ HEPES, 10 EGTA, $2 \mathrm{Mg}$-ATP, $0.5 \quad$ Na2-GTP $\quad(\mathrm{pH}=7.25 ; \quad 295$ mOsm). Voltage-clamp recordings were obtained from whole-cell patch-clamped neurons at room temperature using a Multi-clamp 700B amplifier (Molecular Devices, Sunnyvale, CA, USA), Digidata 1550 Data Acquisition System (Molecular Devices) and a personal computer running pClamp10.7 software. Initial recordings were obtained at $-60 \mathrm{mV}$. Following $120 \mathrm{~s}$ of recording at $60 \mathrm{mV}$, the voltage was incrementally adjusted to +60 $\mathrm{mV}$ to remove blockade of NMDAR responses by magnesium ions. Pharmacological blockers were added following acquisition of a minimum of $20 \mathrm{mEPSC}$ events at $+60 \mathrm{mV}$ in a 10 -min baseline recording period.

\section{Pharmacological inhibition of specific NMDAR GluN2 subunits}

Unless otherwise stated, all chemicals used in this experiment were obtained from Sigma-Aldrich. For GluN2 subtype-specific antagonists, $1 \mu \mathrm{M}$ Ro-25-6981 (Tocris Bioscience) was acutely perfused to selectively inhibit GluN2B-containing NMDARs [14]. It should be noted that NMDAR inhibition by Ro25-6981 is activitydependent [14], which means that NMDAR mEPSC blockade by acute perfusion of $1 \mu \mathrm{M}$ Ro25-6981, as performed here, may underrepresent the fraction of synaptic current that is mediated by GluN2B NMDARs. We applied TCN-201 (Tocris Bioscience) at a concentration $(10 \mu \mathrm{M})$ that robustly blocks GluN2A diheteromeric NMDARs but not GluN2B, GluN2C, or GluN2D diheteromeric NMDARs [15]. The potency of GluN2A
NMDAR blockade by TCN-201 decreases with increasing glycine concentration [15], but with basal glycine concentrations in the rat spinal cord at approximately $13 \mu \mathrm{M}$ [20], application of $10 \mu \mathrm{M}$ TCN-201 will still block approximately $90 \%$ of GluN2A diheteromeric NMDARs. As $10 \mu \mathrm{M}$ TCN-201 has recently been reported to also block over $60 \%$ of triheteromeric NMDARs containing both GluN2A and GluN2B [21], it is possible that some of the TCN-201-sensitive NMDAR current is also mediated by GluN2A/2B triheteromeric NMDARs. The GluN2D (and GluN2C) subunit-specific inhibitor, $10 \mu \mathrm{M}$ DQP-1105 (Tocris Bioscience) [16], was used to assess the contribution of GluN2D-containing NMDARs to synaptic responses in early postnatal lamina II neurons. Each antagonist was dissolved from stock concentrations in DMSO $(10 \mathrm{mM})$ to the final concentrations listed above into bubbled aCSF, without any visible precipitate forming. Antagonists were perfused during recording for an average of $38.2 \pm 1.7 \mathrm{~min}(n=41)$.

\section{Statistical analysis}

All results are presented as average \pm standard error of the mean (SEM). Statistical comparisons of data were performed using the following (where applicable): Student's paired t-test, student's unpaired t-test (unequal variance), and one- or two- way ANOVA followed by a Tukey's test for means comparisons. Results were interpreted as statistically significant at $p<0.05$.

\section{Supplementary information}

Supplementary information accompanies this paper at https://doi.org/10 1186/s13041-020-00566-9.

Additional file 1 Supplementary Fig. 1 Both GluN2B and GluN2A contribute prominently to NMDAR responses at lamina II synapses. Average NMDAR charge transfer following application of 1 uM Ro256981 (red, $n=13$ ) or 10 uM TCN-201 (green, $n=14$ ), with the baseline NMDAR charge transfer for the corresponding cells shown in the black bars to the left. Note the lack of difference between baseline charge transfer values for Ro25-6981- versus TCN-201-treated cells.

\section{Abbreviations}

AMPAR: a-amino-3-hydroxy-5-methyl-4-isoxazolepropionate receptor; mEPSC: miniature excitatory postsynaptic current; NMDAR: N-methyl-Daspartate receptor; Postnatal day 7: P7

\section{Acknowledgements \\ We thank Drs. Melissa Chee and Hongyu Sun for helpful comments on the study and manuscript.}

\section{Authors' contributions}

HM performed all of the electrophysiological experiments, analyzed the majority of the data, prepared the first draft of all figures, and wrote the first draft of the manuscript. NM participated in analysis of electrophysiological datasets and provided feedback on the manuscript text and figures. $\mathrm{MH}$ conceptualized the design and objectives of the study, provided support for experimental data collection and analyses, and was a major contributor in writing the manuscript. The author(s) read and approved the final manuscript. 


\section{Funding}

MH was supported by a John R. Evans Leaders Fund grant from the Canada Foundation for Innovation and an Early Career Research Grant from the International Association for the Study of Pain for experimental equipment and infrastructure required for the study, as well as a Discovery Grant from the Natural Sciences and Engineering Research Council of Canada and an Early Career Investigator Pain Research Grant from the Canadian Pain Society and Pfizer Canada for direct research costs associated with the execution, analysis and publication of the study results.

\section{Availability of data and materials}

All data generated or analysed during this study are included in this published article (and its supplementary information files). Any additional information related to the current study is available from the corresponding author on reasonable request.

\section{Ethics approval and consent to participate}

All rodent experiments followed the guidelines set out by the Canadian Council on Animal Care regarding animal care and handling. Specific animal research protocols were approved by both the Animal Care Committee at Carleton University as well as by the Animal Veterinary Services at The Heart Institute where research animals were housed.

\section{Consent for publication}

Not applicable.

\section{Competing interests}

The authors declare that they have no competing interests.

\section{Author details}

'Department of Neuroscience, Carleton University, Ottawa, ON, Canada. ${ }^{2}$ Neuroscience Program, Ottawa Hospital Research Institute, Ottawa, ON, Canada. ${ }^{3}$ Brain and Mind Research Institute, University of Ottawa, Ottawa, ON, Canada.

Received: 12 November 2019 Accepted: 12 February 2020

Published online: 05 March 2020

\section{References}

1. Todd AJ. Neuronal circuitry for pain processing in the dorsal horn. Nat Rev Neurosci. 2010;11:823-36.

2. Dedek A, Xu J, Kandegedara CM, Lorenzo L-É, Godin AG, De Koninck Y, et al. Loss of STEP61 couples disinhibition to N-methyl-d-aspartate receptor potentiation in rodent and human spinal pain processing. Brain. 2019;142: 1535-46.

3. Bourinet E, Altier C, Hildebrand ME, Trang T, Salter MW, Zamponi GW Calcium-permeable ion channels in pain signaling. Physiol Rev. 2014;94:81140.

4. Paoletti P, Bellone C, Zhou Q. NMDA receptor subunit diversity: impact on receptor properties, synaptic plasticity and disease. Nat Rev Neurosci. 2013; 14:383-400 Available from: http://www.ncbi.nlm.nih.gov/pubmed/23686171.

5. Tong C-K, MacDermott AB. Synaptic GluN2A and GluN2B containing NMDA receptors within the superficial dorsal horn activated following primary afferent stimulation. J Neurosci. 2014;34:10808-20 Available from: http:// www.ncbi.nlm.nih.gov/pubmed/25122884.

6. Bardoni R, Magherini PC, MacDermott AB. NMDA EPSCs at glutamatergic synapses in the spinal cord dorsal horn of the postnatal rat. J Neurosci. 1998;18:6558-67 Available from: http://www.ncbi.nlm.nih.gov/pubmed/96 98343.

7. Hildebrand ME, Pitcher GM, Harding EK, Li H, Beggs S, Salter MW. GluN2B and GluN2D NMDARs dominate synaptic responses in the adult spinal cord. Sci Rep. 2014:4:4094

8. Crair MC, Malenka RC. A critical period for long-term potentiation at thalamocortical synapses. Nature. 1995;375:325-8 Available from: http:// www.ncbi.nlm.nih.gov/pubmed/7753197.

9. Hsia AY, Malenka RC, Nicoll RA. Development of excitatory circuitry in the hippocampus. J Neurophysiol. 1998;79:2013-24 Available from: http://www. ncbi.nlm.nih.gov/pubmed/9535965.

10. Gray JA, Shi Y, Usui H, During MJ, Sakimura K, Nicoll RA. Distinct modes of AMPA receptor suppression at developing synapses by GluN2A and GluN2B: single-cell NMDA receptor subunit deletion in vivo. Neuron. 2011;71:1085-
101 Available from: https://inkinghub.elsevier.com/retrieve/pii/S0896627311 00688X.

11. Dumas TC. Developmental regulation of cognitive abilities: modified composition of a molecular switch turns on associative learning. Prog Neurobiol. 2005;76:189-211 Available from: https://inkinghub.elsevier.com/ retrieve/pii/S0301008205000894.

12. Hildebrand ME, Xu J, Dedek A, Li Y, Sengar AS, Beggs S, et al. Potentiation of synaptic GluN2B NMDAR currents by Fyn kinase is gated through BDNFmediated Disinhibition in spinal pain processing. Cell Rep Elsevier. 2016;17: 2753-65.

13. Fitzgerald $M$, Jennings $E$. The postnatal development of spinal sensory processing. Proc Natl Acad Sci U S A. 1999;96:7719-22.

14. Fischer G, Mutel V, Trube G, Malherbe P, Kew JN, Mohacsi E, et al. Ro 256981, a highly potent and selective blocker of $\mathrm{N}$-methyl-D-aspartate receptors containing the NR2B subunit. Characterization in vitro. J Pharmacol Exp Ther. 1997;283:1285-92 Available from: http://www.ncbi.nIm. nih.gov/pubmed/9400004

15. Hansen KB, Ogden KK, Traynelis SF. Subunit-selective allosteric inhibition of Glycine binding to NMDA receptors. J Neurosci. 2012;32:6197-208 Available from: http://www.ncbi.n/m.nih.gov/pubmed/22553026.

16. Acker TM, Yuan H, Hansen KB, Vance KM, Ogden KK, Jensen HS, et al. Mechanism for noncompetitive inhibition by novel GluN2C/D N -methyl-daspartate receptor subunit-selective modulators. Mol Pharmacol. 2011;80: 782-95 Available from: http://www.ncbi.nlm.nih.gov/pubmed/21807990.

17. Todd AJ. Identifying functional populations among the interneurons in laminae I-III of the spinal dorsal horn. Mol Pain. 2017;13:174480691769300 Available from: http://www.ncbi.nlm.nih.gov/pubmed/28326935.

18. Häring $M$, Zeisel $A$, Hochgerner $H$, Rinwa $P$, Jakobsson JET, Lönnerberg $P$, et al. Neuronal atlas of the dorsal horn defines its architecture and links sensory input to transcriptional cell types. Nat Neurosci. 2018;21:869-80 Available from: http://www.ncbi.nlm.nih.gov/pubmed/29686262.

19. Sathyamurthy A, Johnson KR, Matson KJE, Dobrott Cl, Li L, Ryba AR, et al. Massively parallel single nucleus transcriptional profiling defines spinal cord neurons and their activity during behavior. Cell Rep. 2018;22:2216-25 Available from: http://www.ncbi.nlm.nih.gov/pubmed/29466745.

20. Skilling SR, Smullin DH, Beitz AJ, Larson AA. Extracellular amino acid concentrations in the dorsal spinal cord of freely moving rats following Veratridine and nociceptive stimulation. J Neurochem 1988;51:127-132. Available from: http://doi.wiley.com/https://doi.org/10.1111/j.1471-4159. 1988.tb04845.x.

21. Hansen KB, Ogden KK, Yuan $H$, Traynelis SF. Distinct functional and pharmacological properties of Triheteromeric GluN1/GluN2A/GluN2B NMDA receptors. Neuron. 2014;81:1084-96 Available from: http://www.ncbi.nlm.nih. gov/pubmed/24607230.

\section{Publisher's Note}

Springer Nature remains neutral with regard to jurisdictional claims in published maps and institutional affiliations.
Ready to submit your research? Choose BMC and benefit from:

- fast, convenient online submission

- thorough peer review by experienced researchers in your field

- rapid publication on acceptance

- support for research data, including large and complex data types

- gold Open Access which fosters wider collaboration and increased citations

- maximum visibility for your research: over $100 \mathrm{M}$ website views per year

At $\mathrm{BMC}$, research is always in progress.

Learn more biomedcentral.com/submissions 